\title{
The Centre of the Spaces of Banach Lattice-Valued Continuous Functions on the Generalized Alexandroff Duplicate
}

\author{
Faruk Polat \\ Department of Mathematics, Firat University, 23119 Elazig, Turkey \\ Correspondence should be addressed to Faruk Polat, faruk.polat@gmail.com
}

Received 12 December 2010; Accepted 13 February 2011

Academic Editor: Yong Zhou

Copyright (C) 2011 Faruk Polat. This is an open access article distributed under the Creative Commons Attribution License, which permits unrestricted use, distribution, and reproduction in any medium, provided the original work is properly cited.

We characterize the centre of the Banach lattice of Banach lattice $E$-valued continuous functions on the Alexandroff duplicate of a compact Hausdorff space $K$ in terms of the centre of $C(K, E)$, the space of $E$-valued continuous functions on $K$. We also identify the centre of $C D_{0}(Q, E)=$ $C(Q, E)+c_{0}(Q, E)$ whose elements are the sums of $E$-valued continuous and discrete functions defined on a compact Hausdorff space $Q$ without isolated points, which was given by Alpay and Ercan (2000).

\section{Preliminaries and Definitions}

Throughout the paper, our terminology is mainly standard and a background on Riesz spaces and Banach lattices may be obtained from [1] or [2]. In order to avoid trivial cases, we assume that all topological spaces are nonempty and all Banach lattices are nonzero.

The centre of a Banach lattice $E$, denoted by $Z(E)$, is the lattice of the linear operators, $T: E \rightarrow E$ for which there exists a real number $\lambda>0$ such that $|T x| \leq \lambda|x|$ for all $x \in E$. The operator norm of a central operator $T$ is the minimum of those $\lambda$ with this property. It is well known that $Z(E)$ equipped with the operator norm is an $A M$-space with order unit. The order unit is identity operator $I$.

For a given locally compact Hausdorff space $K$ and a Banach lattice $E, C_{0}(K, E)$ denotes the space of all continuous functions $f$ from $K$ into $E$ which vanish at infinity; that is, there exists a compact set $A \subset K$ such that $\|f(k)\|<\varepsilon$ for each $\varepsilon>0$ and $k \in K \backslash A$. We consider this space to be normed by

$$
\|f\|=\sup \{\|f(k)\|: k \in K\},
$$


and ordered by

$$
f \geq g \Longleftrightarrow f(k) \geq g(k), \quad \forall k \in K
$$

One can show that $C_{0}(K, E)$ is a Banach lattice with these definitions.

Ercan and Wickstead [3] showed that the centre of $C_{0}(K, E)$ is isometrically Riesz isomorhic to $C^{b}\left(K, Z(E)_{s}\right)$ the space of all functions $f$ from $K$ into $Z(E)$ such that $f$ is norm bounded, continuous, and $f\left(k_{\alpha}\right)(e) \rightarrow f(k)(e)$ in $E$ for each $e \in E$ whenever $k_{\alpha} \rightarrow k$ in $K$. Here, $Z(E)$ is given the strong operator topology.

If $K$ is a compact Hausdorff space, then $C_{0}(K, E)=C(K, E)$, where $C(K, E)$ is the space of continuous functions $f: K \rightarrow E$. Hence, the centre of $C(K, E)$ can also be identified with $C^{b}\left(K, Z(E)_{s}\right)$. We will use this identification in the sequel.

If $K$ is a discrete topological space, then $C_{0}(K, E)$ is the space of $E$-valued bounded functions $f$ on $K$ such that the set

$$
\{k \in K: \varepsilon<\|f(k)\|\}
$$

is finite for each $\varepsilon>0$, and we will write $c_{0}(K, E)$ in this case.

Let $\Sigma$ and $\Gamma$ be compact Hausdorff and locally compact Hausdorff topologies on a nonempty set $K$, respectively, such that $\Sigma$ is coarser than $\Gamma$. These topologies on $K$ will be denoted by $K_{\Sigma}$ and $K_{\Gamma}$. The compact Hausdorff topology on $K \times\{0,1\}$ generated by the open base $\mathcal{A}=\mathcal{A}_{1} \cup \mathcal{A}_{2}$, where

$$
\begin{gathered}
\mathcal{A}_{1}=\{H \times\{1\}: H \text { is } \Gamma \text {-open }\}, \\
\mathcal{A}_{2}=\{G \times\{0,1\} \backslash M \times\{1\}: G \text { is } \Sigma \text {-open, } M \text { is } \Gamma \text {-compact }\}
\end{gathered}
$$

is called generalized Alexandroff duplicate of $K$ and denoted by $K_{\Sigma, \Gamma} \otimes\{0,1\}$ (see [4]). When $\Gamma$ is discrete topology on $K$, the compact Hausdorff topological space $K_{\Sigma, \Gamma} \otimes\{0,1\}$ will be denoted by $A(K)$. The space $A(K)$ was first considered by Engelking [5]. For $K=[0,1]$ under the usual metric topology, $A(K)$ was constructed by Alexandroff and Urysohn [6] as an example of a compact Hausdorff space containing a discrete dense subspace. This space is called the Alexandroff duplicate.

Note that $K \times\{0\}$ is a closed subspace of $K_{\Sigma, \Gamma} \otimes\{0,1\}$ and the map $k \rightarrow(k, 0)$ is a homeomorphism between $K_{\Sigma}$ and $K \times\{0\}$.

In [4], it is not proved that $K_{\Sigma, \Gamma} \otimes\{0,1\}$ is a compact Hausdorff space. We give the proof here for the benefit of the reader.

Theorem 1.1. $K_{\Sigma, \Gamma} \otimes\{0,1\}$ is a compact Hausdorff space.

Proof. Consider an open cover $\left\{O_{i}\right\}_{i \in I}$ of $K_{\Sigma, \Gamma} \otimes\{0,1\}$. By replacing each set in the cover by a union of basic open neighborhoods of all points in the set, we can assume that the cover is formed by basic open neighborhoods of the form

$$
\left\{H_{\alpha} \times\{1\}\right\}_{\alpha \in I} \cup\left\{G_{\gamma} \times\{0,1\} \backslash M_{\gamma} \times\{1\}\right\}_{\gamma \in \Omega}
$$


where $H_{\alpha}$ is a $\Gamma$-open set, $G_{\gamma}$ is a $\Sigma$-open set, and $M_{\gamma}$ is a $\Gamma$-compact set. It is easy to see that $\left\{G_{\gamma} \times\{0\}\right\}_{\gamma \in \Omega}$ is an open cover of $K \times\{0\}$, thus there is a finite subcover $G_{\gamma_{1}} \times\{0\}, \ldots, G_{\gamma_{n}} \times\{0\}$. Then,

$$
G_{\gamma_{1}} \times\{0,1\} \backslash M_{\gamma_{1}} \times\{1\} \cup \cdots \cup G_{\gamma_{n}} \times\{0,1\} \backslash M_{\gamma_{n}} \times\{1\}
$$

misses only finitely many $\Gamma$-compact sets $M_{\gamma_{1}} \times\{1\}, \ldots, M_{\gamma_{n}} \times\{1\}$.

As $M_{\gamma_{j}}(j=1,2, \ldots, n)$ is compact, we have that $M_{\gamma_{j}} \times\{1\} \subset \cup H_{\alpha} \times\{1\}$. So, $M_{\gamma_{j}} \times\{1\} \subset$ $\cup_{p=1}^{n} H_{p^{j}} \times\{1\}$. Hence, if we add the corresponding open sets from the cover, then we obtain a finite cover of the entire space $K_{\Sigma, \Gamma} \otimes\{0,1\}$. Therefore, $K_{\Sigma, \Gamma} \otimes\{0,1\}$ is compact.

To show that $K_{\Sigma, \Gamma} \otimes\{0,1\}$ is Hausdorff, it is enough to show that $(k, 0)$ and $(k, 1)$ can be separated. Let $V$ be a $\Gamma$-open neighborhood of $k$ such that $c l_{\Gamma}(V)$ (closure of $V$ in $K_{\Gamma}$ ) is compact. Then, $K_{\Sigma, \Gamma} \otimes\{0,1\} \backslash\left(c l_{\Gamma}(V) \times\{1\}\right)$ and $V \times\{1\}$ are the separating open sets of $(k, 0)$ and $(k, 1)$, respectively. This completes the proof.

If $K_{\Sigma}$ is a compact Hausdorff space without isolated points and $K_{\Gamma}$ is a discrete topological space, then $C\left(K_{\Sigma}, E\right) \cap c_{0}\left(K_{\Gamma}, E\right)=\{0\}$ and $C D_{0}\left(K_{\Sigma}, E\right)=C\left(K_{\Sigma}, E\right) \oplus c_{0}\left(K_{\Gamma}, E\right)$ is a Banach lattice under the pointwise ordering and supremum norm of the sums $f+d$, where $f \in C\left(K_{\Sigma}, E\right)$ and $d \in c_{0}\left(K_{\Gamma}, E\right)$. We refer to [7-9] for more detailed information on these spaces. In [4], it is showed that $C D_{0}\left(K_{\Sigma}, E\right)$ is isometrically Riesz isomorphic to $C(A(K), E)$, where $A(K)$ is the Alexandroff duplicate of $K$. We will use this identification in the sequel to characterize the centre of the space $C D_{0}\left(K_{\Sigma}, E\right)$.

\section{Main Results}

Let $\Sigma$ and $\Gamma$ be compact Hausdorff and locally compact Hausdorff topologies on $K$, respectively, such that $\Sigma$ is coarser than $\Gamma$, and let $E$ be a Banach lattice. Then $C^{b_{*}}\left(K_{\Sigma}, Z(E)_{s}\right)$ denotes the set of all norm bounded and continuous functions $f$ from $K$ into $Z(E)$ such that $r_{\alpha} f\left(k_{\alpha}\right)(e) \rightarrow r f(k)(e)$ in $E$ for each $e \in E$ whenever $\left(k_{\alpha}, r_{\alpha}\right) \rightarrow(k, r)$ in $K_{\Sigma, \Gamma} \otimes\{0,1\}$.

We consider the vector space $C^{b}\left(K_{\Sigma}, Z(E)_{s}\right) \times C^{b_{*}}\left(K_{\Sigma}, Z(E)_{s}\right)$ equipped with coordinatewise algebraic operations, the order

$$
0 \leq(f, d) \Longleftrightarrow 0 \leq f(k)(e), \quad 0 \leq f(k)(e)+d(k)(e) \quad \text { for each } k \in K,
$$

and the norm

$$
\|(f, d)\|=\max \{\|f(k)+r d(k)\|:(k, r) \in K \times\{0,1\}\} .
$$

The norm defined on $C^{b}\left(K_{\Sigma}, Z(E)_{s}\right) \times C^{b_{*}}\left(K_{\Sigma}, Z(E)_{s}\right)$ makes it a Banach space. This is clear, as this norm is equivalent to standard products norms (we have, e.g., $(1 / 2) \max \{\|f\|,\|d\|\} \leq\|(f, d)\| \leq(\|f\|+\|d\|))$. This has no relation to Banach lattices, but it is just a property of Banach spaces. The space $C^{b}\left(K_{\Sigma}, Z(E)_{s}\right) \times C^{b_{*}}\left(K_{\Sigma}, Z(E)_{s}\right)$ is a lattice. This is proved by computing $|(f, d)|=(|f|,|f+d|-|f|)$, where the absolute values on the right-hand side are pointwise. The norm defined on $C^{b}\left(K_{\Sigma}, Z(E)_{s}\right) \times C^{b_{*}}\left(K_{\Sigma}, Z(E)_{s}\right)$ is a Riesz norm. This is obvious from definitions. Therefore, the space $C^{b}\left(K_{\Sigma}, Z(E)_{s}\right) \times C^{b_{*}}\left(K_{\Sigma}, Z(E)_{s}\right)$ is a Banach lattice. 
Actually, the space $C^{b}\left(K_{\Sigma}, Z(E)_{s}\right) \times C^{b_{*}}\left(K_{\Sigma}, Z(E)_{s}\right)$ is isometrically Riesz isomorphic to $C^{b}\left(K_{\Sigma, \Gamma} \otimes\{0,1\}, Z(E)_{s}\right)$ the space of norm bounded, continuous functions $f$ from $K \times\{0,1\}$ into $Z(E)$ such that $f\left(k_{\alpha}, r_{\alpha}\right)(e) \rightarrow f(k, r)(e)$ in $E$ for each $e \in E$ whenever $\left(k_{\alpha}, r_{\alpha}\right) \rightarrow(k, r)$ in $K_{\Sigma, \Gamma} \otimes\{0,1\}$ as the following shows.

Theorem 2.1. $C^{b}\left(K_{\Sigma}, Z(E)_{s}\right) \times C^{b_{*}}\left(K_{\Sigma}, Z(E)_{s}\right)$ and $C^{b}\left(K_{\Sigma, \Gamma} \otimes\{0,1\}, Z(E)_{s}\right)$ are isometrically Ries isomorphic spaces.

Proof. Define the map

$$
\pi: C^{b}\left(K_{\Sigma}, Z(E)_{s}\right) \times C^{b_{*}}\left(K_{\Sigma}, Z(E)_{s}\right) \longrightarrow C^{b}\left(K_{\Sigma, \Gamma} \otimes\{0,1\}, Z(E)_{s}\right),
$$

by

$$
\pi(f, d)(k, r)(e)=f(k)(e)+r d(k)(e),
$$

for each $(k, r) \in K \times\{0,1\}$ and $e \in E$.

Let $\left(k_{\alpha}, r_{\alpha}\right) \rightarrow(k, r)$ in $K_{\Sigma, \Gamma} \otimes\{0,1\}$. Then, $k_{\alpha} \rightarrow k$ in $K_{\Sigma}$ so that $f\left(k_{\alpha}\right)(e) \rightarrow f(k)(e)$ and $r_{\alpha} d\left(k_{\alpha}\right)(e) \rightarrow r d(k)(e)$ in $E$ for each $e \in E$. Hence, $f\left(k_{\alpha}\right)(e)+r_{\alpha} d\left(k_{\alpha}\right)(e) \rightarrow f(k)(e)+$ $r d(k)(e)$ in $E$ for each $e \in E$ so that the map $\pi$ is well defined. It follows immediately that $\pi$ is an isometry, as $\pi(f, d)$ agrees with $f+d$ on $K \times\{1\}$ and with $f$ on $K \times\{0\}$. It is obvious that $\pi(f, d) \geq 0 \Leftrightarrow(f, d) \geq 0$.

It remains to show that $\pi$ is onto. Let $h \in C^{b}\left(K_{\Sigma, \Gamma} \otimes\{0,1\}, Z(E)_{s}\right)$ be given. Define

$$
f(k)(e)=h(k, 0)(e), \quad d(k)(e)=h(k, 1)(e)-h(k, 0)(e),
$$

for each $k \in K$ and $e \in E$. The norm boundedness of $f$ and $d$ follows directly from the norm boundedness of $h$. If $k_{\alpha} \rightarrow k$ in $K_{\Sigma}$, then $\left(k_{\alpha}, 0\right) \rightarrow(k, 0)$ in $K_{\Sigma, \Gamma} \otimes\{0,1\}$ so that

$$
f\left(k_{\alpha}\right)(e)=h\left(k_{\alpha}, 0\right)(e) \longrightarrow h(k, 0)(e)=f(k)(e),
$$

in $E$ for each $e \in E$, hence $f \in C^{b}\left(K_{\Sigma}, Z(E)_{s}\right)$.

To show that $d \in C^{b_{*}}\left(K_{\Sigma}, Z(E)_{s}\right)$, let $\left(k_{\alpha}, r_{\alpha}\right) \rightarrow(k, r) \in K_{\Sigma, \Gamma} \otimes\{0,1\}$. We now examine the possibilities.

Suppose first that $r=1$. Then, $\left(r_{\alpha}\right)$ is eventually 1 . As $\left(k_{\alpha}, 0\right) \rightarrow(k, 0)$ in $K_{\Sigma, \Gamma} \otimes\{0,1\}$, we have $r_{\alpha} d\left(k_{\alpha}\right)(e) \rightarrow r d(k)(e)$ in $E$ for each $e \in E$ in this possibility.

Suppose now that $\left(k_{\alpha}, r_{\alpha}\right) \rightarrow(k, 0)$ and assume that $r_{\alpha} d\left(k_{\alpha}\right)(e)$ does not converge to zero in $E$. Then, there is a subnet $\left(r_{\alpha_{\beta}}\right)$ of $\left(r_{\alpha}\right)$ such that $r_{\alpha_{\beta}}=1$ and $\varepsilon<\left\|d\left(k_{\alpha_{\beta}}\right)(e)\right\|$ for each $\beta$ and for some $\varepsilon>0$. On the other hand, since $\left(k_{\alpha_{\beta}}, 1\right) \rightarrow(k, 0)$ and $\left(k_{\alpha_{\beta}}, 0\right) \rightarrow(k, 0)$ in $K_{\Sigma, \Gamma} \otimes$ $\{0,1\}$, we have $h\left(k_{\alpha_{\beta}}, 1\right)(e) \rightarrow h(k, 0)(e)$ and $h\left(k_{\alpha_{\beta}}, 0\right)(e) \rightarrow h(k, 0)(e)$ so that $d\left(k_{\alpha_{\beta}}\right)(e)=$ $h\left(k_{\alpha_{\beta}}, 1\right)(e)-h\left(k_{\alpha_{\beta}}, 0\right)(e) \rightarrow 0$. This contradiction shows that $d \in C^{b_{*}}\left(K_{\Sigma}, Z(E)_{s}\right)$. It is clear that $\pi(f, d)=h$, and this completes the proof.

Since $Z\left(C\left(K_{\Sigma}, E\right)\right)$ and $Z\left(C\left(K_{\Sigma, \Gamma} \otimes\{0,1\}, E\right)\right)$ can be identified with $C^{b}\left(K_{\Sigma}, Z(E)_{s}\right)$ and $C^{b}\left(K_{\Sigma, \Gamma} \otimes\{0,1\}, Z(E)_{s}\right)$, respectively, we immediately have the following from the previous theorem. 
Corollary 2.2. $Z\left(C\left(K_{\Sigma, \Gamma} \otimes\{0,1\}, E\right)\right.$ and $Z\left(C\left(K_{\Sigma}, E\right)\right) \times C^{b_{*}}\left(K_{\Sigma}, Z(E)_{s}\right)$ are isometrically Riesz isomorphic spaces.

Let $K_{\Gamma}$ be a discrete topology, and let $E$ be a Banach lattice. The set of all bounded functions $f: K \rightarrow Z(E)$ such that the set $\{k: \varepsilon<\|f(k)(e)\|$ for all $e \in E\}$ is finite will be denoted by $c_{0}\left(K_{\Gamma}, Z(E)_{s}\right)$.

Lemma 2.3. Let $K_{\Sigma}$ be a compact Hausdorff space, and let $\Gamma$ be a discrete topology on $K$. Then, $C^{b_{*}}\left(K_{\Sigma}, Z(E)_{s}\right)=c_{0}\left(K_{\Gamma}, Z(E)_{s}\right)$.

Proof. Let $f \in c_{0}\left(K_{\Gamma}, Z(E)_{s}\right)$. Suppose that $f \notin C^{b_{*}}\left(K_{\Sigma}, Z(E)_{s}\right)$. Then, there exists a net $\left(k_{\alpha}, 1\right)$ in $A(K)$ such that $\left(k_{\alpha}, 1\right) \rightarrow(k, 0) \in A(K)$ and $\varepsilon<\left\|f\left(k_{\alpha_{\beta}}\right)(e)\right\|$ for some subnet $\left(k_{\alpha_{\beta}}\right)$ of $\left(k_{\alpha}\right)$, $\varepsilon>0$, and for each $e \in E$. So, $\left(k_{\alpha_{\beta}}\right)$ has finite range which is a contradiction. Conversely, assume that $f \in C^{b_{*}}\left(K_{\Sigma}, Z(E)_{s}\right)$ but $f \notin c_{0}\left(K_{\Gamma}, Z(E)_{s}\right)$. Then, there exist some $e \in E$ and a sequence $\left(k_{n}\right)$ such that $\varepsilon<\left\|f\left(k_{n}\right)(e)\right\|$ for each $n$ and $k_{n} \neq k_{m}$ whenever $n \neq m$. Then, there exists a subnet $\left(k_{n_{\alpha}}\right)$ of $k_{n}$ such that $\left(k_{n_{\alpha}}, 1\right) \rightarrow(k, 0)$ so that $f\left(k_{n_{\alpha}}\right)(e) \rightarrow 0$ which is impossible and this completes the proof.

By Theorem 2.1 and the previous lemma, we have the following.

Theorem 2.4. Let $K_{\Sigma}$ be a compact Hausdorff space, and let $\Gamma$ be a discrete topology on $K$. Then, $C^{b}\left(A(K), Z(E)_{s}\right)$ and $C^{b}\left(K_{\Sigma}, Z(E)_{s}\right) \times c_{0}\left(K_{\Gamma}, Z(E)_{s}\right)$ are isometrically Riesz isomorphic spaces.

As the centre of $C D_{0}\left(K_{\Sigma}, E\right)$ can be identified with $C^{b}\left(A(K), Z(E)_{s}\right)$, we immediately have Theorem 3.1 of [8] as follows.

Corollary 2.5. Let $K_{\Sigma}$ be a compact Hausdorff space without isolated points, and let $\Gamma$ be a discrete topology on $K$. Then, the centre of $C D_{0}\left(K_{\Sigma}, E\right)$ and $Z\left(C\left(K_{\Sigma}, E\right)\right) \times c_{0}\left(K_{\Gamma}, Z(E)_{s}\right)$ are isometrically Riesz isomorphic spaces.

Note that in the corollary above, if all the operators $T \in Z(E)$ are norm attaining; that is, there exists some $e \in E$ with $\|e\|=1$ such that $\|T\|=\|T(e)\|$, then $c_{0}\left(K_{\Gamma}, Z(E)_{s}\right)$ can be replaced by $c_{0}\left(K_{\Gamma}, Z(E)\right)$.

\section{References}

[1] C. D. Aliprantis and O. Burkinshaw, Positive Operators, Springer, Dordrecht, The Netherlands, 2006.

[2] P. Meyer-Nieberg, Banach Lattices, Universitext, Springer, Berlin, Germany, 1991.

[3] Z. Ercan and A. W. Wickstead, "Banach lattices of continuous Banach lattice-valued functions," Journal of Mathematical Analysis and Applications, vol. 198, no. 1, pp. 121-136, 1996.

[4] M. Çaglar, Z. Ercan, and F. Polat, "Generalized Alexandroff duplicates and $C D_{0}(K)$ spaces," Central European Journal of Mathematics, vol. 4, no. 3, pp. 371-375, 2006.

[5] R. Engelking, "On the double circumference of Alexandroff," Bulletin de l'Académie Polonaise des Sciences, vol. 16, pp. 629-634, 1968.

[6] P. S. Alexandroff and P. S. Urysohn, "Memoire sur les espaces topologiques compacts," Koninklijke Nederlandse Akademie van Wetenschappen te Amsterdam, vol. 14, pp. 1-96, 1929.

[7] Y. A. Abramovich and A. W. Wickstead, "Remarkable classes of unital AM-spaces," Journal of Mathematical Analysis and Applications, vol. 180, no. 2, pp. 398-411, 1993.

[8] S.. Alpay and Z. Ercan, "C $C D_{0}(Q, E)$ and $C D_{\omega}(Q, E)$-spaces as Banach lattices," Positivity, vol. 4, no. 3, pp. 213-225, 2000.

[9] Z. Ercan, "A concrete description of $C D_{0}(K)$-spaces as $C(X)$-spaces and its applications," Proceedings of the American Mathematical Society, vol. 132, no. 6, pp. 1761-1763, 2004. 


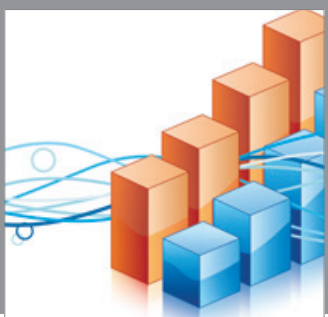

Advances in

Operations Research

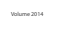

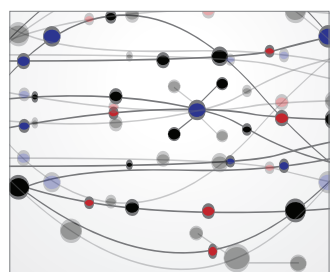

\section{The Scientific} World Journal
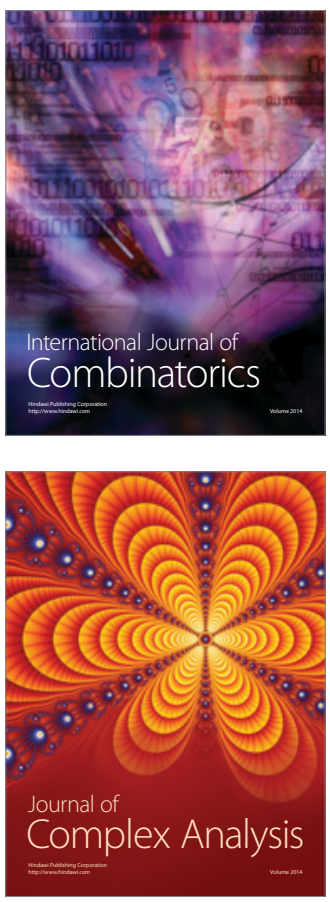

International Journal of

Mathematics and

Mathematical

Sciences
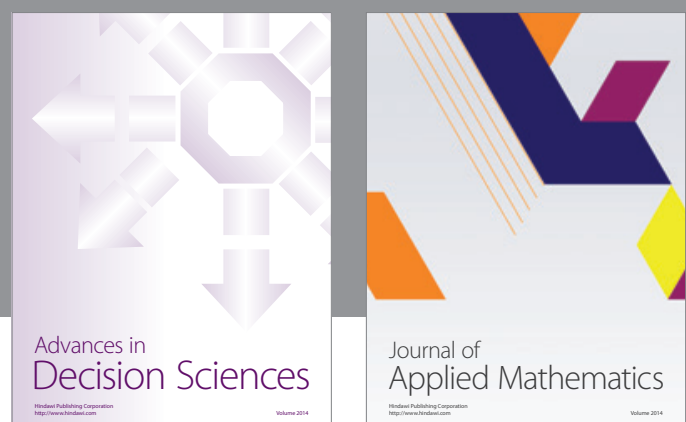

Journal of

Applied Mathematics
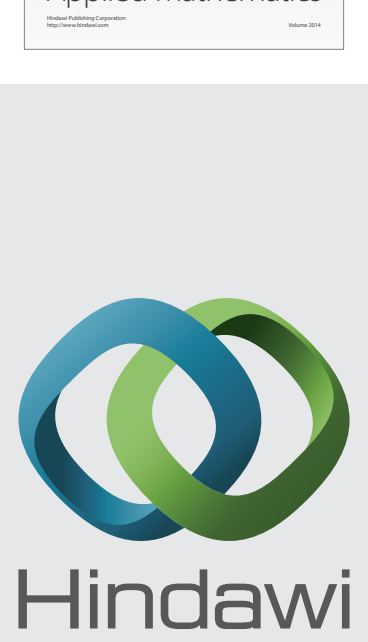

Submit your manuscripts at http://www.hindawi.com
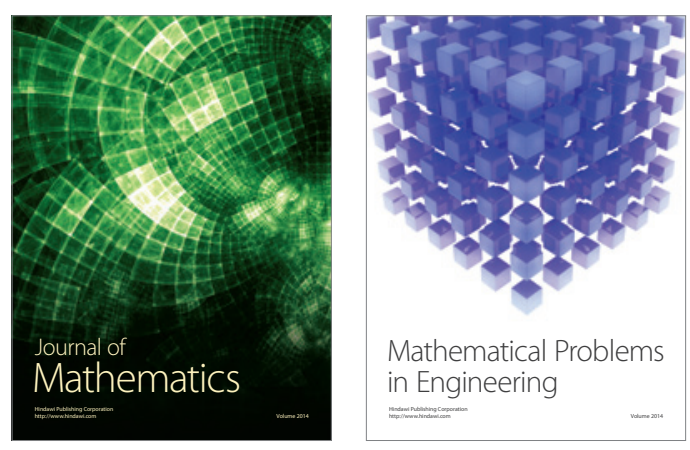

Mathematical Problems in Engineering
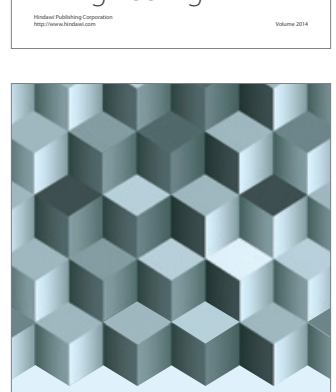

Journal of

Function Spaces
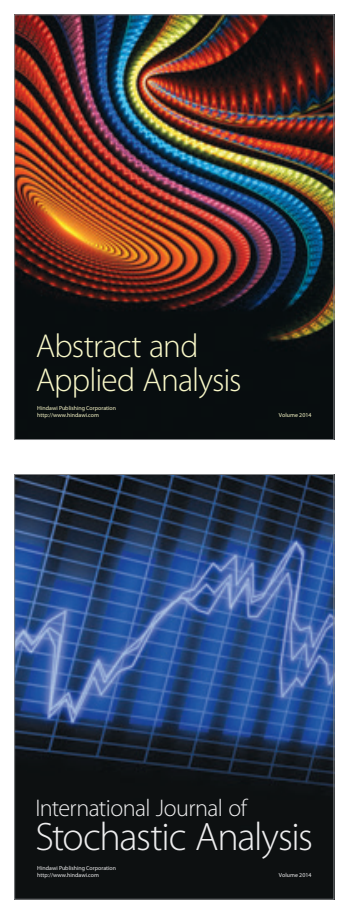

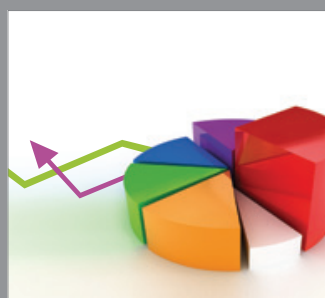

ournal of

Probability and Statistics

Promensencen
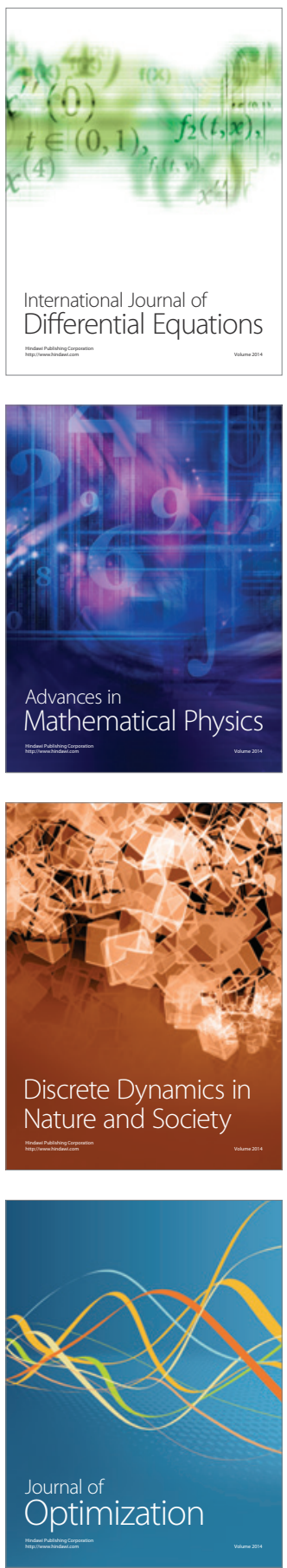